\title{
A literature Review of the Results from Nursing and Psychosocial Research within Swedish Pediatric Oncology
}

Karin Enskär*, Susanne Knutsson, Karina Huus, Mats Granlund, Laura Darcy and Maria Björk

School of Health Sciences, Jönköping University, Sweden Child research group, Jönköping, Sweden.

*Corresponding author: Karin Enskär, School of Health Sciences, Jönköping University, Sweden Child research group, Jönköping, Sweden, Email:karin.enskar@hhj.hj.se

Received date: September 11, 2014, Accepted date: November 18, 2014, Publication date: November 21, 2014

Copyright:@ 2014 Enskär K, et al. This is an open-access article distributed under the terms of the Creative Commons Attribution License, which permits unrestricted use, distribution, and reproduction in any medium, provided the original author and source are credited.

\begin{abstract}
The body of research-based knowledge in paediatric caring science has been increasing leading to dramatic improvements in treatment. The purpose of this manuscript was to analyze results as stated by the researchers', in recently published articles on nursing and psychosocial research, within Swedish pediatric oncology setting. This was done through a review of 137 published articles about paediatric oncology related to caring science in Sweden. The result shows that the illness has affected, in both positive and negative ways, the wellbeing of everyone coming into contact with the child. The cancer also causes distress related to all aspects of life including physical, psychological, existential and social. Mediating factors for the experience of distress and wellbeing are: disease and treatment severity, age, gender and ethnicity of the participant, time since diagnosis, the use of internal and external support, and the identity of the person reporting the data. Health promoting aspects frequently reported are: family togetherness, coping strategies, engaging in normal life and activities, and quality of care which includes emotional support, information and family participation in care. The hospital staff has to be aware of the psychosocial issues experienced by children with cancer and their families, and they have to acknowledge the value of formal interventions, reporting benefits for children, families, and themselves.
\end{abstract}

Keywords: Cancer; Childhood; Caring science; Literature review; Results; Sweden

\section{Introduction}

Over the past 40 years, 5-years survival rates of children and adolescents with cancer have risen dramatically in Sweden as well as in other countries, an improvement largely attributed to increasingly effective treatment [1]. However more can be done to improve both survival rates and quality of survival [2]. Nursing science and psychosocial science, hereafter named caring science, has long played a central role in paediatric oncology clinical services and research. The body of research-based knowledge in oncology caring science has increased dramatically. Early work focused on symptom relief, related to the side effects of chemotherapy, and pain management related to invasive medical procedures. As survival rates improved, the focus has shifted to descriptive studies examining the psychosocial impact on children and their families during and after treatment [3].

Despite the growing number of research reports on the impact of childhood cancer, there are still few published studies describing the children's own perspectives on living with cancer. Wakefield et al. [4] carried out a literature review on psychosocial functioning of children who had recently completed cancer treatment, and found that they may experience positive psychosocial outcomes on treatment completion, including improved self-worth, improved mental health and social behaviour. However, they may also experience significant negative outcomes, including lower levels of psychological well-being, mood, liveliness, self-esteem, and motor and physical functioning, as well as increased anxiety, problem behaviours and sleeping difficulties.

Young adult survivors of childhood cancer are a growing population of patients who may have a lifelong risk of potential complications resulting from their cancer therapy; these can have a negative effect on their health and wellbeing [5-6]. On the whole, the adjustments of young cancer survivors as a group are reasonably good, but the findings with respect to emotional and social adjustment are inconsistent [7-8]. According to Patenaude and Kupst [9], low or average levels of distress are found in survivors of paediatric cancer; however there are also subsets of more vulnerable patients. A literature review on qualitative studies identified seven important areas of wellbeing: physical, social, psychological, spiritual and fertility/sexual wellbeing, resilience and body appearance [10].

Parents' wellbeing related to the child's cancer has been widely studied. However, published studies do not agree on the significance of the negative psychological impact on them. Parents can experience significant distress, including fear of recurrence, fatigue, loneliness [11] anxiety, depression, prolonged grief or poor quality of life [12]. Others claim that parents and other family-members adjust well and that only a minority appear to be at risk of psychological morbidity [13-14]. Research has proven that positive aspects of wellbeing, such as self-esteem and mastery, are important predictors for parents' wellbeing as well as their view of the child's prognosis [15].

Siblings also experience negative effects on their wellbeing as a result of a sister's or brother's cancer [16]. Reported symptoms in siblings are: anxiety, negative emotional reactions, behavioural problems, poor social adjustment self-esteem, and post-traumatic stress symptoms (17-18). However, Buchbinder et al. [19] found that siblings of childhood cancer survivors were generally psychologically healthy.

In a literature review by Knops et al.[20], 131 recommendations were made for care for children with cancer. An expert panel reduced the list to 109 , including 31 prioritized recommendations. The 
prioritized care recommendations were formed into five categories: Medical care, Communication, Education and training, General quality measures, and Structural facilities of care. Caring issues were sparsely included in those recommendations, even though several authors have pointed out the need for structured psychosocial care [3,21-22] and quality nursing care [23] as well as benefits of such interventions [24]. Patenaude and Kupst [9] reviewed research on psychosocial paediatric oncology research, and found that in the past 30 years paediatric oncology care has gone through changes from avoidance of communication about cancer to an emphasis on straightforward discussion of the illness and prognosis. Research has shown the benefits of information/education for children and adolescents [25-26], and for parents [27].

Over the next five years, according to the Children's Oncology Group's 2013 blueprint for research, the field of nursing and psychosocial science needs to translate empirical research into practice $[3,23]$. This requires that research is solid and based clinically relevant issues, and that staff use research to continue to evolve knowledge within paediatric oncology caring [28].

\section{Aim}

The purpose of this manuscript was to analyze results as stated by the researchers', in recently published articles on nursing and psychosocial research, within Swedish pediatric oncology setting.

\section{Methods}

\section{Study Design}

This study presents a systematic literature review based on a mixedmethod approach $[29,30]$ performed in steps. The selection of studies was performed in four steps:

- A database search using keywords as well as inclusion and exclusion criteria was performed to identify and select articles.

- The entire articles were reviewed on a full-text level using a mapping protocol.

- The content of the results included in the articles were analyzed using content analysis.

- The categories and subcategories from the content analysis was compared to other variables from the mapping protocol.

\section{The Setting of Swedish Pediatric Oncology}

Every year, approximately 300 children in Sweden are diagnosed with cancer; some 80 percent survive, thanks to major advances in research and in care and treatment over the past forty years. The treatment of cancer in children has improved considerably over the last decade, especially in regards to the subsequent quality of life of these children after treatment. In Sweden, paediatric oncology units have been centralized into six treatment centres to meet medical and nursing care demands for children suffering from cancer. The costs of treatment and care are covered by the government and follow international harmonized treatment protocols and standards. It is common for parents to stay with their child in the hospital and in the home, thanks to a generous national insurance system in Sweden. National collaborative networks are organized between nurses, social workers and psychologists, for quality of life improvement.
Researchers in Sweden have carried out extensive research in nursing and psychosocial care during the last three decades, in comparison to other countries primarily as a result of grants from The Swedish Childhood Cancer Foundation, which sponsors most of this research (www.barncancerfonden.se).

\section{Literature Search}

In the first step, studies relevant to caring science presenting empirical data from Swedish participants were identified through a search in the databases CINAHL, Psyc INFO, and PubMed. Keywords used in the search were selected to identify articles which could fit the selection criteria for the study: adolescent OR child OR children OR childhood OR pediatric; AND "Stem Cell Transplantation" OR cancer OR neoplasm OR oncolog*; AND "home care" OR holistic OR "quality of life" OR psychosocial OR social OR bereave ${ }^{*}$ OR psycholog* OR nurs ${ }^{\star}$; AND Sweden; AND English; AND NOT (review [pt]).

For inclusion in the literature review, the studies needed to fit the following criteria: subjects diagnosed with cancer before 18 years of age; subjects undergoing or post treatment; a caring science perspective; research carried out in Sweden; and articles written in English and published in a peer-reviewed journal between January 2000 and June 2013. 325 studies were identified through this search, of which 140 were selected by reviewing the titles and abstracts to ensure included articles met the selection criteria. A manual search was carried out based on authors' names using the same databases, as well as online and via authors' webpages in order to identify further possibly relevant studies. First or second authors with more than two publications were included in this subsequent selection process, which yielded another 37 studies. Each of these 177 studies was read and reviewed in its entirety. During the review process, another 40 studies were excluded as they did not fulfil the inclusion criteria: no focus on pediatric patients (16 studies); no focus on Swedish patients and/or their families (8 studies); review articles only (6 studies); no focus on pediatric oncology (5 studies); no caring science perspective (3 studies); and a focus on instrument development (2 studies). All of the remaining 137 studies were included in the analysis.

\section{Review of the Articles}

In the second step, a mapping protocol was developed which was inspired by the McMaster Critical Review Form for qualitative and quantitative studies (31). The protocol allowed for evaluating many aspects of the publications a) the target person of the research, b) the aim, c) scientific tradition and theoretical aspects, d) data collection, e) study design, f) results, g) clinical implication, h) ethics, and i) research funds, as well as $\mathrm{j}$ ) information about the publication such as journal and year. A summary of the most important results, conclusions, and suggestions for clinical implications was also included in the protocol. The mapping of the articles showed that most of the studies were descriptive or comparative and were quantitative in their methodology. Most of them focused on parents (43\%) or children (28\%). Most of the studies investigated wellbeing (88\%), using questionnaires (53\%) or interviews (38\%). The key attributes of the included articles are presented in Table 1 and 2. 
Citation: Enskär KM, Knutsson S, Huus K, Granlund M, Darcy L (2014) A literature Review of the Results from Nursing and Psychosocial Research within Swedish Pediatric Oncology. J Nurs Care 3: 217. doi:10.4172/2167-1168.1000217

Page 3 of 11

\begin{tabular}{|c|c|c|c|c|c|}
\hline & $\mathrm{n}$ & $\%$ & & $\mathrm{n}$ & $\%$ \\
\hline Research Design & & & Participants/Subjects & & \\
\hline Quantitative descriptive design & 104 & 76 & Child (0-18) & 19 & 14 \\
\hline Longitudinal studies & 29 & 21 & Survivors & 15 & 11 \\
\hline Comparisons with health controls (or other diseases) & 24 & 17 & Parents & 55 & 40 \\
\hline Comparisons within the group & 23 & 17 & Siblings & 5 & 4 \\
\hline Cross-sectional studies & 22 & 16 & Nurses & 6 & 4 \\
\hline Intervention study; & 6 & 4 & Physicians & 3 & 2 \\
\hline with control group (as RCT) & 4 & 3 & Data from quality-registrar & 5 & 4 \\
\hline Qualitative design, as; & 48 & 35 & More than one group of participants & 30 & 22 \\
\hline Content analysis & 31 & 23 & Others & 3 & 2 \\
\hline Phenomenology & 9 & 7 & Diagnosis/Stage & & \\
\hline Grounded Theory & 6 & 4 & Mixed diagnoses/stages & 101 & 74 \\
\hline Ethnology & 1 & 1 & Transplantation & 14 & 10 \\
\hline Discourse analysis & 1 & 1 & Palliative phase & 10 & 7 \\
\hline Type of data collection & & & CNS-tumours & 10 & 7 \\
\hline Questionnaire & 73 & 53 & Hematological diseases, incl. leukemia & 1 & 1 \\
\hline Interview (face-to-face) & 33 & 24 & Other specific diagnosis & 1 & 1 \\
\hline Interview (telephone) & 9 & 7 & Age of the child & & \\
\hline Interview using questionnaire & 3 & 2 & No specific age $(0-18$ years old $)$ & 94 & 69 \\
\hline Focus groups interview & 7 & 5 & Survivors & 17 & 12 \\
\hline Observation & 3 & 2 & Adolescents (13-18 years old) & 14 & 10 \\
\hline Data from quality-registrar & 6 & 4 & School-age children (7-12 years old) & 9 & 4 \\
\hline Another sources & 4 & 3 & Pre-school children (0-6 years old) & 3 & 2 \\
\hline \multicolumn{6}{|l|}{ Number of participants/study } \\
\hline$<10$ & 14 & 10 & & & \\
\hline $10-20$ & 17 & 12 & & & \\
\hline $21-50$ & 25 & 18 & & & \\
\hline $51-100$ & 21 & 15 & & & \\
\hline $101-500$ & 44 & 32 & & & \\
\hline$>500$ & 16 & 12 & & & \\
\hline
\end{tabular}

Table 1: Presentation of type of studies included in the review.

\begin{tabular}{|c|c|c|c|c|c|c|}
\hline $\begin{array}{l}\text { Focus of the research/ } \\
\text { Target person/s }\end{array}$ & $\mathrm{N}(\%)$ & Children $(n=39)$ & $\begin{array}{l}\text { Survivors } \\
(\mathrm{n}=17)\end{array}$ & $\begin{array}{l}\text { Parents } \\
(\mathrm{n}=59)\end{array}$ & $\begin{array}{l}\text { Siblings } \\
(n=6)\end{array}$ & Staff/care $(n=16)$ \\
\hline $\begin{array}{l}\text { Wellbeing; as } \\
\text { Experiences, Quality of Life (QoL), Anxiety or } \\
\text { other Psychosocial problems }\end{array}$ & $\begin{array}{l}121 \\
88 \%\end{array}$ & 39 & 14 & 55 & 5 & 8 \\
\hline
\end{tabular}




\begin{tabular}{|l|l|l|l|l|l|l|}
\hline $\begin{array}{l}\text { Caring aspects; as } \\
\text { General and Organization of care, Palliative } \\
\text { care and Information }\end{array}$ & $\begin{array}{l}39 \\
28 \%\end{array}$ & 10 & 2 & 20 & 1 & 6 \\
\hline $\begin{array}{l}\text { Specific areas; as } \\
\text { Pain or Nutrition }\end{array}$ & $\begin{array}{l}27 \\
20 \%\end{array}$ & 10 & 5 & 8 & 4 \\
\hline $\begin{array}{l}\text { Social life; as } \\
\text { Relationships with Family members and Friends } \\
\text { and Social support }\end{array}$ & $\begin{array}{l}20 \\
15 \%\end{array}$ & 3 & 2 & 13 & 2 & \\
\hline
\end{tabular}

Table 2: The focus of the studies included in the review.

A detailed presentation of the result from the mapping protocol has been reported elsewhere (32).

\section{Content Analysis}

In the third step, the reported results in each of the 137 articles were inductively analyzed by the first author (KE) in collaboration with all authors, using inductive manifest content analysis as described by Elo and Kyngäs [33]. This revealed 791 different statements comprising result of the studies, with 1-19 statements per article (median 3-5 statements per article). These 791 statements were first assigned codes which were organized by content into different sub-categories. These subcategories were analyzed and reorganized into 20 categories based on similarities and differences in content. The categories were analyzed again and reorganized into four categories based on similarities and differences in content (Table 3). The continuous movement back and forth between the codes, subcategories, and categories gained a trustworthy result. Collaboration with coresearchers added to the study's reliability and validity.

\begin{tabular}{|l|l|l|l|l|l|l|l|}
\hline General wellbeing & Total & Child $\mathbf{n}=\mathbf{3 9}$ & Survivor $\mathbf{n = 1 7}$ & Parents $\mathbf{n = 5 9}$ & Siblings $\mathbf{n}=\mathbf{6}$ & Staff $\mathbf{n}=\mathbf{7}$ & Care $\mathbf{n}=\mathbf{9}$ \\
\hline Effects on wellbeing & 19 & 6 & 3 & 8 & - & 1 & 1 \\
\hline Good wellbeing & 19 & 5 & 7 & 4 & 1 & 1 & 1 \\
\hline Not good wellbeing & 26 & 9 & 3 & 2 & - & - \\
\hline
\end{tabular}

Table 3: Frequency of articles mention wellbeing related to the research participant.

\section{Relating Categories to the Mapping Protocol}

In the fourth step, the categories and subcategories from the content analysis (step 3) was entered into the data file together with all other variables from the mapping-protocol. This open for a possibility of carrying out comparison between the qualitative and the quantitative results, a mixt-method approach [29,30]. Descriptive statistics as numbers and percentages were used.

\section{Results}

This review shows that the illness affects, both positively and negatively, the wellbeing of everyone coming in contact with the child. It also causes distress related to all aspects of life including physical, psychological, existential and social. Mediating factors for decreasing distress and increasing wellbeing are disease and treatment severity, age, gender and ethnicity of the individual, time since diagnosis, the use of internal and external support, as well as who reported the data. Frequently reported health promoting factors are family togetherness, having coping strategies and engaging in the activities of normal life, as well as quality of care: emotional support, information and family participation in care.

\section{The child's illness has consequences for the wellbeing of the child and of the children network}

The results show $47 \%(65 / 137)$ of the articles pointing out that the illness has affected the wellbeing of the ill child as well as all those coming in contact with the child (as parents, siblings and staff) (Table 3).

The results are not conclusive and give a divided picture with both positive and negative effects on the wellbeing of children and families. The consequences could be described as generally negative $(n=6)$ or as positive $(\mathrm{n}=10)$, as "All the negative and difficult experiences are compensated for with a positive view on and positive expectations regarding life" ( $p u b 33$ ). The duality of both positive and negative consequences was often stated for children and some articles $(n=13)$ describe it as a transition for the family from having a healthy child to a new everyday life dealing with a sick child, treatment, side-effects and hospitalization "The cancer disease and its treatment affected several aspects of everyday life of these parents" (pub 92). A further 20 statements showed that the wellbeing can be considered as good $(\mathrm{n}=20)$ or not worse compared to the general population $(\mathrm{n}=10)$, "However, neither the current status in the different areas nor the QoL index score differed between survivors and controls."(pub 132). This was most often seen in studies on survivors. However, others claim wellbeing to be bad $(n=24)$ or worse than the general population $(n=14)$, especially in relation to that of parents. 
For health care staff, caring for children with cancer leads to personal $(\mathrm{n}=2)$ and professional $(\mathrm{n}=2)$ development "The vast majority $(76,7 \%)$ stated that working in this medical field was very stimulating for their personal development" (pub 114).

In three studies the authors stated that the participants did not report negative distress of participating in research. The articles also stated that aspects of wellbeing as quality of life QoL $(n=3)$ or stress $(\mathrm{n}=2)$ can be measured "The results support the construct validity of the disease specific SEIQOL-DW and indicate that the instrument appears to be a sensitive measure ... (pub 136).

\section{Experiences of distress related to the child's illness}

The research showed that the child's illness causes distress for the child as well as for others coming in contact with the child; as parents, siblings and staff. This distress is related to all aspects of life including physical, psychological, existential and social (Table 4).

\begin{tabular}{|l|l|l|l|l|l|l|l|}
\hline Distress & Total & $\begin{array}{l}\text { Child } \\
\mathrm{n}=39\end{array}$ & Survivor $\mathrm{n}=17$ & Parents $\mathrm{n}=59$ & Siblingsn=6 & $\begin{array}{l}\text { Staffn=7 } \\
\mathrm{n}=9\end{array}$ \\
\hline Physical distress & 42 & 20 & 9 & 9 & 1 & - \\
\hline Pain & 13 & 8 & - & 2 & - & 3 \\
\hline Tiered, sleep, vitality & 10 & 5 & - & 3 & 1 & - & - \\
\hline Psychological distress & 64 & 21 & 5 & 35 & 1 & 11 & 1 \\
\hline Psychological distress (general) & 22 & 9 & 2 & 11 & - & - & - \\
\hline Worries, fear, anxiety & 30 & 11 & 2 & 17 & - & 13 & - \\
\hline Mood, sadness, depression, PTS & 18 & 4 & 1 & - & - & - \\
\hline Existential distress & 6 & 2 & - & 2 & - & 1 \\
\hline Social distress & 18 & 3 & 3 & 10 & 1 & - \\
\hline
\end{tabular}

Table 4: Frequency of articles mention distress and being related to the research participant.

Physical distress $(\mathrm{n}=42 / 31 \%)$ is described by general statements $(\mathrm{n}=6)$ relating to children and also to parents "...poor physical health" (pub 61). The most commonly mentioned physical distress is pain $(\mathrm{n}=13)$, general pain, procedure related, treatment related, disease related and headache, "... pain resulting from diagnostic procedures and treatments..." (pub 49). Other commonly mentioned physical problems were: energy level $(\mathrm{n}=10)$ as tiredness, problem sleeping and low vitality "The most frequently reported physical problem was fatigue." (pub 35), late effects of treatment and need for rehabilitation $(n=8)$, problems with eating $(n=4)$, such as nausea, taking medication and food aversion, hair loss, mucositis, skin-related symptoms $(n=4)$, body changes such as mobility problems $(n=3)$, cognitive $(n=3)$ or sexual problems $(n=1)$.

Psychological distress $(n=64 / 47 \%)$ is described by general statements about physical distress $(\mathrm{n}=35)$ in parents, but also in children "...who describe themselves as suffering severe mental distress which they relate to their illness and treatment" (pub47). The most frequently mentioned psychological distress was worry $(\mathrm{n}=31)$ "The most prominent fear of mothers and fathers of CNS tumor patients is that of a recurrence of the tumor...", fear "...expressing fear were being quite ...” (pub 8), and anxiety”...many parents experience high levels of disease-related stress even after successful treatment." (pub 95). The reason for this distress was related to medical procedures, insecurity, side-effects, relapse, death and the future. The other psychological problems mentioned were mood changes including sadness, depression, and PTS $(\mathrm{n}=18)$ "...mothers reported that they had been bothered by general fatigue..., depression....and headache...fathers reported... general fatigue..., depression... and headache..." (pub 131), heavy emotional burden and crisis $(n=9)$, loneliness and isolation $(n=8)$, and changed self-image $(\mathrm{n}=8)$.

In a few articles $(n=6)$ existential distress was addressed. Being forced to conduct medical procedures with children not noncollaborating children $(n=3)$ was considered stressful as it was for staff to bring bad news to parents $(\mathrm{n}=1)$ "...being a messenger of lifethreatening conditions...” (pub 113). Other mentioned aspects of existential pain were: existential extortion and hopelessness.

Social distress $(n=18 / 13 \%)$ was most often related to parents' life such as changed work situation $(n=7)$, effect on family finances $(n=6)$, need for knowledge $(n=5)$, negative effects on family life and friends $(n=4)$, being dependent on the care $(n=2)$, and general social distress $(n=1)$. Children and survivors mentioned negative effects on school and education $(\mathrm{n}=3)$, and the social distress could be exemplified as, "In both groups, family life, relations to other people work and carrier, interests and leisure activities were areas most frequently reported to influence quality of life." (pub 116).

\section{The child's and family's situation are experienced differently by different people and during different phases of the cancer trajectory}

The children and the family members experience different aspect of wellbeing and distress during different phases of the illness. Factors mediating the experience of distress and wellbeing were disease and treatment, gender, ethnicity, age, internal and external support as well as the identity of the reporting person (Table 5). 
Citation: Enskär KM, Knutsson S, Huus K, Granlund M, Darcy L (2014) A literature Review of the Results from Nursing and Psychosocial Research within Swedish Pediatric Oncology. J Nurs Care 3: 217. doi:10.4172/2167-1168.1000217

Page 6 of 11

\begin{tabular}{|c|c|c|c|c|c|c|c|}
\hline Mediating factors & Total & Child $n=39$ & $\begin{array}{l}\text { Survivor } \\
\mathrm{n}=17\end{array}$ & Parents $n=59$ & Siblings $n=6$ & Staffn=7 & $\begin{array}{l}\text { Care } \\
n=9\end{array}$ \\
\hline \multicolumn{8}{|l|}{ Diagnose, prognosis, treatment } \\
\hline Differences between diagnoses & 20 & 2 & 8 & 8 & 2 & - & \\
\hline No diff. between diagnoses & 3 & 1 & 1 & 1 & - & - & \\
\hline \multicolumn{8}{|l|}{ Frequency of symptoms } \\
\hline Differences related to symptoms & 16 & 7 & 1 & 8 & - & - & \\
\hline \multicolumn{8}{|l|}{ Time after diagnose, treatment } \\
\hline Different during different phases & 19 & 6 & 1 & 12 & - & - & \\
\hline No diff. during different phases & 4 & 2 & - & 2 & - & - & \\
\hline \multicolumn{8}{|l|}{ Gender } \\
\hline Gender differences & 20 & 1 & 5 & 13 & - & 1 & \\
\hline No gender differences & 2 & 1 & - & 1 & - & - & \\
\hline \multicolumn{8}{|l|}{ Age of the children } \\
\hline Age differences & 9 & 6 & 1 & 1 & - & - & 1 \\
\hline \multicolumn{8}{|l|}{ Ethnicity } \\
\hline Ethnicity differences & 5 & - & - & 3 & - & - & 2 \\
\hline \multicolumn{8}{|l|}{ Internal support } \\
\hline Inner capacity is useful: & 9 & 2 & - & 6 & - & 1 & \\
\hline \multicolumn{8}{|l|}{ External support, health care } \\
\hline External support is useful & 13 & 2 & - & 8 & 1 & 1 & 1 \\
\hline \multicolumn{8}{|l|}{ Who is answering } \\
\hline Diff. - who is answering & 19 & 8 & 1 & 6 & 1 & 1 & 2 \\
\hline No diff. - who is answering & 1 & 1 & - & - & - & - & \\
\hline
\end{tabular}

Table 5: Mediating factors related to the research participant.

Some diseases and treatments $(n=20)$ were more often associated with increased distress such as CNS tumours $(n=7)$, loss of the child $(\mathrm{n}=5)$, intensive treatment $(\mathrm{n}=3)$, bad prognosis $(\mathrm{n}=2)$, and transplantation $(\mathrm{n}=1)$, "CNS tumours and combined treatment where somewhat associated to a higher extent of negative consequences." (pub 119). Other authors ( $\mathrm{n}=3$ ) did not find any differences between diagnoses or treatment groups. However, having a high prevalence of symptoms was associated with reduced wellbeing $(n=16)$.

In 20 of the studies gender differences were investigated. The authors found that females (mothers and girls) were more effected by the illness than males $(\mathrm{n}=15)$ "...girls rating worse HRQOL compared with boys." (pub 1), except for one $(\mathrm{n}=1)$ study showing that male survivors have more sexual problems than female ones "The results indicate that cancer disease and treatment have more impact on sexual function on male survivors than on the sexual function of female survivors." (pub 117). Other studies report gender differences but do not specify them $(n=7)$, and four reported that they did not find gender differences.
In five studies ethnicity was studied. Four studies found immigrant parents to be more effected by the child's illness "The pattern of stress symptoms may vary according to educational level, ethnicity and gender." (pub 95), and one study found that Swedish parents had a different level of wellbeing from that of parents from another countries.

In nine studies the age of the child was addressed and the results were not conclusive. Two found no age differences and seven stated that there were age differences but that no pattern could be found. "Children who died at 9-15 years of age were reported to be moderately or severely affected, by a number of symptoms, significantly more often than other children." (pub 59).

In the included articles, distress appears to decline, and wellbeing to increase, over time even though these changes might take a long time. Two studies stated that the distress was different in different phases of the illness and two that there were no differences between the different phases. In 19 studies wellbeing increased over time "...lower among parents for whom a longer period of time had elapsed from the time of 
diagnosis." (pub 17), in three no differences were seen between the phases, and in two studies distress increased over time. This was mostly seen in studies with parents. In 15 of the studies it was stated that the decrease of distress takes a long time "About one fifth of parents reported psychological and financial difficulties exceeding the cut off limit for a significant impact still > 5 years after diagnosis" (pub 57).

In this review internal support $(\mathrm{n}=9)$ such as personality, socialnetwork and education level seems to mediate the experience of distress and wellbeing in children "Self-reported HRQOL was positively correlated to days of school attendance." (pub 1), and in parents. Also external support $(n=13)$ such as information, professional support, and health care interventions can decrease distress and increase wellbeing, "...the children experienced the relation with the hospital clowns, how they described the magical aspects of the encounter and how they viewed the importance of clown encounters to their own well-being." (pub 77).

In 20 of the studies researchers investigated whether different people would report the same degrees of distress and wellbeing. In 19 of these studies differences were found related to who reported the data "Both physicians and nurses overestimated levels of anxiety and depression." (pub 50) and in one study no differences were found.

\section{The childrens' and families' situations can be enhanced by internal and external support}

The children and families use the support provided to ease the impact of the illness on their lives. The most frequently mentioned health promoting factors for parents were social support, family togetherness and use of coping strategies. For children a "normal" life, and being able to participate in activities, were also mentioned (Table 6). Aspects of social support often mentioned were given by family $(n=23)$ and friends $(n=12)$, the child's school $(n=4)$, practical and economical support $(\mathrm{n}=5)$, and support from other parents with children with cancer $(n=1)$. Family togetherness was also often mentioned for example having a family life, being together "Closeness with other people, especially their own family, was important" (pub 13), paying attention to all family members including siblings, and parenting aspects such as being fair. Another health promoting activity was effective use of coping strategies which could help the child and family to deal with the situation, "...most adolescents reported using emotion-focused coping (Accepting and Minimizing) while ....meaning-based (i.e. Positive thinking) and problem-focused (i.e. Problem solving) coping were most often mentioned" (pub 31). Being at home, living a "normal life" was something everyone wished to do, "Patients who were treated at home enjoyed being active and taking a walk when they felt like it" (pub 123). Keeping up and being engaged in activities such as play, school, work, friends, leisure and physical activities was described as helpful. The same was stated for keeping up relationships “...meeting friends...” (pub 36).

The quality of care was also described as promoting health and wellbeing in the children and their families. In four of the studies good general care was mentioned as important (Table 4). For parents the most often mentioned aspects of care were support, information and family participation. For children, specific interventions and a caring approach were also often mentioned. The most frequently mentioned aspect was information. Children and their families not only need information, the need for quality of information has also been studied. The information needs to be easy assessable, correct, well-timed and based on individual preferences "Matching the amount of information to parents needs concerned situations where the amount of information provided according to the care givers assessment is deemed too small, appropriate, or to large" (pub 108). Another often mentioned aspect of good care was high quality support from health care professionals "...had access to psychological support during the last month of their child's life...were more likely to have worked through their grief" (pub 68). Support was most often mentioned as emotional support, communication or just "support". A few articles stated that professional support should be offered to all family member, not forgetting siblings $(\mathrm{n}=2)$, "A therapeutic support group for siblings of children with cancer is beneficial. ...the groups helpful in coping with their situation" (pub 87) or by psychologist $(\mathrm{n}=2)$. Others mention that the support could be given in groups or through the web. Other important areas were family participation in care, staff willingness to attend to developmental and individual needs as well as the staff having a caring approach "Important care for adolescents treated for cancer consists mainly of meeting nice friendly, supportive, and competent staff..." (pub 52), the staff being knowledgeable and willing to collaborate $(n=13)$, hospitals having a caring environment $(\mathrm{n}=12)$; including the possibility of play, attending to childrens' needs, a free zone for children, and time and $(n=10)$. A further 13 studies presented specific interventions $(n=13)$ to be used in paediatric oncology care, two interventions related to nutrition and the rest to pain and anxiety in children "...non-immersive $V R$ (virtual reality) is a positive experience for children undergoing a minor procedure..." (pub 86).

\begin{tabular}{|c|c|c|c|c|c|c|c|}
\hline & $\begin{array}{l}\text { Tot } \\
\text { al }\end{array}$ & $\begin{array}{l}\text { Childn } \\
=39\end{array}$ & $\begin{array}{l}\text { Survivo } \\
\mathrm{r} n=17\end{array}$ & $\begin{array}{l}\text { Parent } \\
\text { s n=59 }\end{array}$ & $\begin{array}{l}\text { Sibling } \\
\text { sn=6 }\end{array}$ & $\begin{array}{l}\text { Staffn } \\
=7\end{array}$ & $\begin{array}{l}\text { Care } \\
n=9\end{array}$ \\
\hline $\begin{array}{l}\text { Health } \\
\text { promoting } \\
\text { factors }\end{array}$ & 45 & 13 & 6 & 17 & 4 & 2 & 3 \\
\hline Support & 17 & 5 & 1 & 7 & 2 & 1 & 1 \\
\hline $\begin{array}{l}\text { Coping } \\
\text { strategies }\end{array}$ & 15 & 7 & 2 & 3 & - & 2 & 1 \\
\hline Family & 13 & 3 & - & 6 & 2 & - & 2 \\
\hline $\begin{array}{l}\text { Engagement in } \\
\text { activities }\end{array}$ & 10 & 4 & 3 & 1 & 1 & - & 1 \\
\hline $\begin{array}{l}\text { Living a "normal } \\
\text { life" }\end{array}$ & 9 & 2 & 1 & 2 & 2 & 1 & 1 \\
\hline Quality of care & 53 & 13 & 2 & 22 & 4 & 4 & 8 \\
\hline $\begin{array}{l}\text { Professional } \\
\text { support }\end{array}$ & 21 & 5 & 1 & 11 & 4 & - & - \\
\hline Information & 15 & 3 & 1 & 8 & 2 & 1 & - \\
\hline $\begin{array}{l}\text { Family } \\
\text { participation }\end{array}$ & 12 & 3 & - & 5 & 1 & - & 3 \\
\hline $\begin{array}{lr}\text { Listen } & \text { to } \\
\text { individual needs }\end{array}$ & 11 & 2 & - & 4 & - & 1 & 4 \\
\hline $\begin{array}{l}\text { Caring } \\
\text { approach }\end{array}$ & 9 & 4 & - & 1 & 1 & 2 & 1 \\
\hline
\end{tabular}




\begin{tabular}{|l|l|l|l|l|l|l|l|}
\hline $\begin{array}{l}\text { Specific } \\
\text { interventions }\end{array}$ & 7 & 5 & 1 & - & - & 1 & - \\
\hline
\end{tabular}

Table 6: Internal and external support related to the research participant.

\section{Discussion}

This review shows that the illness affects the wellbeing of everyone coming in contact with the child. It also causes distress related to all aspects of life. Mediating factors for decreasing distress and increasing wellbeing are disease and treatment severity, age, gender and ethnicity of the individual, time since diagnosis, the use of internal and external support, as well as who reported the data. Frequently reported health promoting factors are family togetherness, having coping strategies and engaging in the activities of normal life, as well as quality of care: emotional support, information and family participation in care.

The results show that cancer effects wellbeing. Children and survivors seem to cope with the illness in both a positive and negative way and their wellbeing and quality of life are, in most studies, reported to be similar to those of the healthy population. This is also confirmed by several study's [4, 7-9], who all reported the adjustment of children, and young cancer survivors as reasonably good. However, there are always some patients who are more vulnerable and do not cope well or who have significant difficulties regarding family, social life and personal aspects [9].

A more complex picture was reported among parents of children with cancer. Even as some studies included in this review reported parents to have good wellbeing and quality of life, others reported them to have a negatively affected wellbeing and quality of life. Wakefield et al. [11] stated that parents can experience negative wellbeing while others claimed that parents/family-member adjust well to the cancer, and that only a minority appears to be at risk of psychological morbidity $[13,14]$. There does not seem to be a consensus between the different studies concerning parents' wellbeing and quality of life. This may have a natural explanation as these concepts are not thoroughly defined [32]. Other concepts such as social support, investigated for families of children with cancer, also seem to lack consensus [34]. Another explanation for the different outcomes could be that researchers use a great variety of methods and measurement instruments [32]. Mattsson et al. [35] showed in their review the positive consequences of childhood cancer and how different study designs bring out different results for the same phenomena. A review by Enskär et al. [32] found that different measurement instruments had been used in each and every study. To get comparable and consistent results in the future, the researchers have to agree on the use of methods, especially instruments to measure wellbeing and quality of life in children, survivors, parents and siblings.

The result of this review also shows that the child's illness should be cause distress related to all aspects of life including physical, psychological, existential and social distress. For children the most commonly reported distress was psychological, manifest as anxiety, followed by physical distress such as pain and tiredness. Wakefield et al. [4] also found in their literature review that distress was related to the cancer experience as increased anxiety, behaviour problems and sleeping difficulties. The management of procedure-related pain has been an important area to investigate for many years since children treated for cancer often report feelings of anxiety and distress [32].
Patenaude and Kupst (9) reported in their review that research has led to interventions to reduce procedure-related distress in children. In the review of Enskär et al. [32] only a few specific interventions were carried out. Those interventions were related to specific areas of the child's care such as management of anxiety as well as pain related to procedures or nutrition.

For the parents in this review, the most often reported distress were psychological distress manifest as worries and anxiety, followed by mood changes such as depression. Those aspects of parents' wellbeing have been studied also outside Sweden. Klassen et al. [36], found that parents of children with cancer reported poorer physical and psychosocial quality of life in all psychosocial domains and in most physical health domains. Others have reported worries, fear of recurrence [11], anxiety, depression and prolonged grief [12].

In this review it was clear that different mediating factors influenced the experience of distress and wellbeing and were not experienced over time in the same way by all those involved. Differences in experience depending on diagnosis, treatment or prognosis could be seen in some studies. Some diagnoses, such as CNS-tumours, long and more advanced treatment protocols, frequency of symptoms and a bad prognosis were all associated with a more negative wellbeing. Langeveld et al. [7] found that demographic, illness and treatment related variables are related to survivors' quality of life. Klassen et al. [36] found in their review that demographic differences, cancer type and treatment regimens all had a significant negative impact on patients' quality of life. Children with lower treatment intensity and higher wellbeing were associated with higher quality of life in the parents. Another mediating factor was time. In this review the distress was different in different phases of the illness; for example the wellbeing increased over time, but it often took a long time. Fakhre et al. [37] carried out a literature review on quality of life from diagnosis to remission/survivorship and end of life for children with cancer. They found that those who are newly diagnosed with cancer and undergo treatment, or are terminally ill, have impaired health-related quality of life (HRQoL). However, survivors of childhood cancer have high HRQoL (with the exception of those who experienced medical comorbidity or posttraumatic stress disorder). Klassen et al. [36] and Wakefield et al. [11] stated that distress in parents appears to ease with time.

In this review the age of the child, as a mediating factor, was not fully clear. Eiser et al. [38] argue that the impact of cancer in young adults is different from experiences during childhood. Key developmental tasks include negotiation of independence from the nuclear family, achievement of intimacy, and generatively (concern to establish and guide the next generation) and all of these tasks can be challenged by cancer, and have led to concern about health-related quality of life. However, as most studies in this review were carried out among parents the differences between the age-groups was mostly seen related to the parents' wellbeing and not the childrens'.

More obvious in this review was the gender differences in adolescents and parents. Gender differences, especially among parents, have been studied and reported [39-40], also traditional gender roles in the division of parental tasks has been reported [40].

The presence of positive intrapsychological traits, such as selfesteem and mastery, was more predictive of parental optimism than other mediating factors, such as prognosis [15], related to the illness. For parents, caregiver strain, self-perception and family-centered services provision was associated with psychosocial wellbeing [41]. 
Furthermore, physical aspects such as better eating, exercise and sleeping habits in parents have been associated with better parental QoL [36]. Wakefield et al. [4] carried out a literature review on psychosocial functioning of children who had recently completed cancer treatment, and found that they may experience self-worth, good behaviourand improved mental health and social skills.

In this review health promoting activities, such as family togetherness, coping strategies and engaging in activities and "normal life", have been reported as important. When a family member becomes ill maintaining daily routines and rituals is an important contributor to retaining stability. The family members can also feel increased cohesiveness within the family, which is perceived as helpful [42].

Peek and Melnyk [24], found in their literature review, that numerous studies have demonstrated the adverse impact of cancer on family members, but few are intervention studies designed to facilitate coping and wellbeing. Eiser et al. [38] state that there is a need for intervention studies, and attempts to improve knowledge, reintegration into normal life and to promote self-care, are described. Pedro et al. [34], investigated social support and they recommend the need for it to be assessed and included in care plans and guidelines. Research has found that family-based psychosocial interventions are feasible, acceptable, and potentially effective to for use in paediatric oncology [43].

In this review the qualities of care comprise emotional support, information and family participation in care. The staff need to have, as well as the staff having a caring approach including listening to the childrens' and parents' needs, and to providing a child centred care. It is important that hospital staff are aware of the psychosocial issues experienced by children with cancer and their families. They recognized the value of formal intervention, reporting benefits for children and, families, and for themselves [44]. Several of the expected behaviours in the staff (the caring approach) could be described using the Swanson Caring Theory [45] which includes: (a) respecting families and believing in their capacity to make the best decisions for their family UNIT (maintaining belief); (b) understanding families' experiences and their continued need to protect their child (knowing); (c) physically and emotionally engaging with the family (being with); (d) providing unbiased information describing all possibilities (enabling); and (e) helping families navigate the system and creating a therapeutic environment for them in which to make decisions (doing for) [46].

According to the Children's Oncology Group's 2013 blueprint for research, over the next 5 years, the field of nursing and psychosocial science needs to translate empirical supported research into practice $[3,23]$. As most studies in this review are descriptive or comparative [32], this is not an easy task for clinical staff. Caring sciences must focus more on intervention studies and longitudinal studies and proportionally less on descriptive research. There is an agreement, that paediatric oncology caring practice must incorporate both the science and the art of the discipline to foster positive physical and psychosocial treatment outcomes for paediatric oncology patients [47].

\section{Limitation of the study}

This systematic review has some limitations. Firstly, the inclusion criteria were only for Swedish studies (published written in English), published between January 2000 and March 2013 and in certain databases. Of course this aims at a more national perspective in paediatric oncology caring sciences rather than giving the full picture of international research. Despite the wide range of keywords in combination with the manual search, it is possible that some potential studies were missed. The second limitation of this study was that no quality review of the included articles was performed prior to the analysis process as it this was deemed unnecessary due to the aim of this review and to the fact that all of the studies had undergone a peer review process prior to being published. The third limitation is related to the fact that most of the studies were descriptive or comparative, focusing on parents' wellbeing [32], which may drive the qualitative results in a certain direction. The fourth limitation is related to the qualitative analyse process as the first author (KE) carried out the main part of the analysis, which could be influenced, by earlier experiences and preconceptions. However, the credibility of the results is strengthened by the fact that data analysis was done rigorously and discussed among the authors during the process. The fifth limitation is that the result in this review is based on the authors' own summary of the results in the articles. This might have had an effect on the number of articles in each presented category. The true number in each category can only be confirmed by doing a new analysis by using the categories (and subcategories) as a mapping protocol. This needs to be carried out in the future.

\section{Conclusion and Recommendations}

This review of the literature has demonstrated that the child's illness has affected, in both positives and negative ways, the wellbeing of all everyone coming into contact with the them, in both positives and negative ways. Also the child's illness causes distress related to all aspects of life including physical, psychological, existential and social distress. Mediating factors for the experience of distress and wellbeing are; disease and treatment severity, age, gender and ethnicity of the participant, time since diagnosis, the use of internal and external support, as well as who the identity of those who answered the questions. Frequent reported health promoting factors are: family togetherness, coping strategies and engaging in activities and of normal life. The family's wellbeing can also be enhanced due to through different aspects of care such as emotional support, information and family participation in care. There is an inconsistency regarding the effect of the illness. Therefore, researchers have to agree on the use of methods, especially instruments, to measure wellbeing and quality of life in children, survivors and families.

There is an urgent need to translate empirical research into practice. As most studies in caring sciences are descriptive or comparative, there is a need for intervention studies and longitudinal studies and proportionally less on descriptive research. There is an agreement, that paediatric oncology caring practice must incorporate both the science and the art of the discipline to foster positive physical and psychosocial treatment outcomes for paediatric oncology patients.

The hospital staffs have to be aware of the psychosocial issues experienced by children with cancer and their families. They have to recognize the value of formal interventions, reporting benefits for children, families, and themselves.

\section{References}

1. Gustafsson G, Heyman M, Vernby $\AA$ (2007) Childhood cancer incidence and survival in Sweden 1984-2005. Stockholm, Sweden: Barncancer Epidemiologiska Forsknings Enheten vid Karolinska Institutet, 
2. Reaman GH (2004) Pediatric cancer research from past successes through collaboration to future transdisciplinary research. J Pediatr Oncol Nurs 21: 123-127.

3. Noll R, Patel S, Embry L, Hardy K, Pelletier W, et al., (2013) COG behavioral Sciences Committee Children's Oncology Group's 2013 blueprint for research: behavioral science. Pediatr Blood Cancer 60(6): 1048-54.

4. Wakefield CE, McLoone J, Goodenough B, Lenthen K, Cairns DR, et al. (2010) The psychosocial impact of completing childhood cancer treatment: a systematic review of the literature. J Pediatr Psychol 35: 262-274.

5. Armenian SH, Landier W, Hudson MM, Robison LL, Bhatia S; COG Survivorship and Outcomes Committee (2013) Children's Oncology Group's 2013 blueprint for research: survivorship and outcomes. Pediatr Blood Cancer 60: 1063-1068.

6. Mulrooney DA, Neglia JP, Hudson MM (2008) Caring for adult survivors of childhood cancer. Curr Treat Options Oncol 9: 51-66.

7. Langeveld NE, Stam H, Grootenhuis MA, Last BF (2002) Quality of life in young adult survivors of childhood cancer. Support Care Cancer 10: $579-600$.

8. Stam H, Grootenhuis MA, Last BF (2001) Social and emotional adjustment in young survivors of childhood cancer. Support Care Cancer 9: 489-513.

9. Patenaude AF, Kupst MJ (2005) Psychosocial functioning in pediatric cancer. J Pediatr Psychol 30: 9-27.

10. Nightingale CL, Quinn GP, Shenkman EA, Curbow BA, Zebrack BJ, et al. (2011) Health-Related Quality of Life of Young Adult Survivors of Childhood Cancer: A Review of Qualitative Studies. J Adolesc Young Adult Oncol 1: 124-132.

11. Wakefield CE, McLoone JK, Butow P, Lenthen K, Cohn RJ (2011) Parental adjustment to the completion of their child's cancer treatment. Pediatr Blood Cancer 56: 524-531.

12. Rosenberg AR, Baker KS, Syrjala K, Wolfe J (2012) Systematic review of psychosocial morbidities among bereaved parents of children with cancer. Pediatr Blood Cancer 58: 503-512.

13. Hagedoorn M, Kreicbergs U, Appel C (2011) Coping with cancer: The perspective of patients' relatives. ActaOncol 50: 205-211.

14. Long KA, Marsland AL (2011) Family adjustment to childhood cancer: a systematic review. Clin Child FamPsychol Rev 14: 57-88.

15. Fayed N, Klassen AF, Dix D, Klaassen R, Sung L (2011) Exploring predictors of optimism among parents of children with cancer. Psychooncology 20: 411-418.

16. Murray JS (1999) Siblings of children with cancer: a review of the literature. J PediatrOncolNurs 16: 25-34.

17. Alderfer MA, Long KA, Lown EA, Marsland AL, Ostrowski NL, et al. (2010) Psychosocial adjustment of siblings of children with cancer: a systematic review. Psychooncology 19: 789-805.

18. Prchal A, Landolt MA (2009) Psychological interventions with siblings of pediatric cancer patients: a systematic review. Psychooncology 18: 1241-1251.

19. Buchbinder D, Casillas J, Krull KR, Goodman P, Leisenring W, et al. (2011) Psychological outcomes of siblings of cancer survivors: a report from the Childhood Cancer Survivor Study. Psychooncology 20: 1259-1268.

20. Knops RR, Hulscher ME, Hermens RP, Hilbink-Smolders M, Loeffen JL, et al. (2012) High-quality care for all children with cancer. Ann Oncol 23: 1906-1911.

21. Askins MA, Moore BD 3rd (2008) Psychosocial support of the pediatric cancer patient: lessons learned over the past 50 years. CurrOncol Rep 10: 469-476.

22. Kusch M, Labouvie H, Ladisch V, Fleischhack G, Bode U (2000) Structuring psychosocial care in pediatric oncology. Patient EducCouns 40: 231-245.
23. Landier W, Leonard M, Ruccione KS (2013) Children's Oncology Group's 2013 blueprint for research: nursing discipline. Pediatr Blood Cancer 60: 1031-1036.

24. Peek G, Melnyk BM (2010) Coping interventions for parents of children newly diagnosed with cancer: an evidence review with implications for clinical practice and future research. PediatrNurs 36: 306-313.

25. Scott JT, Harmsen M, Prictor MJ, Sowden AJ, Watt I (2003) Interventions for improving communication with children and adolescents about their cancer. Cochrane Database Syst Rev (3):CD002969. Review. Update in: Cochrane Database Syst Rev 2008: CD002969.

26. Ranmal R, Prictor M, Scott JT (2008) Interventions for improving communication with children and adolescents about their cancer. Cochrane Database Syst Rev 8:CD002969.

27. Aburn G, Gott M (2011) Education given to parents of children newly diagnosed with acute lymphoblastic leukemia: a narrative review. J Pediatr Oncol Nurs 28: 300-305.

28. Bryant R, Rodgers C, Stone S (2013) Enhancing pediatric oncology nursing care through research, quality improvement, and evidence-based practice. J Pediatr Oncol Nurs 30: 123-128.

29. Creswell JW (2009) Research design: qualitative, quantitative, and mixed methods approaches. Thousand Oaks, Calif., Sage.

30. Tashakkori A, Teddlie C (1998) Mixed methodology: Combining qualitative and quantitative approaches, Thousand Oaks, Calif. SAGE.

31. Letts L, Wilkins S, Law M, Stewart D, Bosch J, et al. (2012) McMaster University Critical Review Guidelines for Qualitative and Quantitative Studies

32. Enskär K, Björk M, Knutsson S, Granlund M, Darcy L, Huus K (2014) A Swedish perspective on nursing and psychosocial research in pediatric oncology: A litterateur review.

33. Elo S, Kyngäs H (2008) The qualitative content analysis process. J Adv Nurs 62: 107-115.

34. Pedro IC, Galvão CM, Rocha SM, Nascimento LC (2008) Social support and families of children with cancer: an integrative review. Rev Lat Am Enfermagem 16: 477-483.

35. Mattsson E, Lindgren B, Von Essen L (2008) Are there any positive consequences of childhood cancer? A review of the literature. Acta Oncol 47: 199-206

36. Klassen AF, Klaassen R, Dix D, Pritchard S, Yanofsky R, et al. (2008) Impact of caring for a child with cancer on parents' health-related quality of life. J Clin Oncol 26: 5884-5889.

37. Fakhry H, Goldenberg M, Sayer G, Aye SS, Bagot K, et al. (2013) Healthrelated quality of life in childhood cancer. J Dev Behav Pediatr 34: 419-440.

38. Eiser C, Penn A, Katz E, Barr R (2009) Psychosocial issues and quality of life. Semin Oncol 36: 275-280.

39. da Silva FM, Jacob E, Nascimento LC (2010) Impact of childhood cancer on parents' relationships: an integrative review. J NursScholarsh 42: 250-261.

40. Clarke NE, McCarthy MC, Downie P, Ashley DM, Anderson VA (2009) Gender differences in the psychosocial experience of parents of children with cancer: a review of the literature. Psychooncology 18: 907-915.

41. Klassen AF, Raina P, McIntosh C, Sung L, Klaassen RJ, et al. (2011) Parents of children with cancer: which factors explain differences in health-related quality of life. Int J Cancer 129: 1190-1198.

42. Patterson JM (2002) Understanding family resilience. J Clin Psychol 58: 233-246.

43. Meyler E, Guerin S, Kiernan G, Breatnach F (2010) Review of familybased psychosocial interventions for childhood cancer. J Pediatr Psychol 35: 1116-1132.

44. Kiernan G, Meyler E, Guerin S (2010) Psychosocial issues and care in pediatric oncology: medical and nursing professionals' perceptions. Cancer Nurs 33: E12-20.

45. Swanson KM (1991) Empirical development of a middle range theory of caring. Nurs Res 40: 161-166. 
Citation: Enskär KM, Knutsson S, Huus K, Granlund M, Darcy L (2014) A literature Review of the Results from Nursing and Psychosocial Research within Swedish Pediatric Oncology. J Nurs Care 3: 217. doi:10.4172/2167-1168.1000217

Page 11 of 11

46. Kavanaugh K, Roscigno CI, Swanson KM, Savage TA, Kimura RE, et al (2013) Perinatal palliative care: Parent perceptions of caring in interactions surrounding counseling for risk of delivering an extremely premature infant.Palliat Support Care 4:1-11.
47. Cantrell MA (2007) The art of pediatric oncology nursing practice. J Pediatr Oncol Nurs 24: 132-138. 